\title{
Shape invariance and SUSY separation of variables
}

\author{
M.V. Ioffe ${ }^{1 a}$, E.V. Kolevatova ${ }^{1 b}$, and V. Vereshagin ${ }^{1 c}$ \\ ${ }^{1}$ Saint-Petersburg State University, 198504 St.-Petersburg, Russia
}

\begin{abstract}
The main ingredients of conventional Supersymmetrical Quantum Mechanics (SUSY QM) are presented. The generalization with supercharges of second order in derivatives - Second Order SUSY - is formulated, and the property of shape invariance is defined. The generalization to two-dimensional coordinate space, after using just these two elements of the modern SUSY QM approach, provides the opportunity to solve analytically some two-dimensional problems. Two different procedures of supersymmetrical separation of variables are formulated. They are illustrated by two-dimensional generalization of the Morse model.
\end{abstract}

\section{Introduction}

The idea of Supersymmetry (SUSY) appeared in the framework of Elementary Particle Theory and Quantum Field Theory in the papers [1]. The main motivation was to extend the Poincare algebra by nontrivial unification of space-time and internal symmetries, i.e. to overcome the limitation of "no-go theorem" [2]. New symmetry was named "super" because of its several exclusive physical properties: unification of internal symmetries with space-time symmetries; new kind of algebra which includes both commutators and anticommutators; inclusion of bosons and fermions into the same multiplets.

One-dimensional SUSY QM was proposed by E.Witten [3] as a toy model of $(0+1)$ Quantum Field Theory which was useful to study the problems of spontaneous breaking of SUSY. The corresponding Lagrangian is:

$$
L=\dot{x}^{2}(t)-\left[\left(W^{\prime}(x(t))^{2}-W^{\prime \prime}(x(t))\right]-2 W^{\prime \prime}(x(t)) \psi^{+} \psi^{-},\right.
$$

where $x$ is a coordinate, $\psi^{ \pm}$are fermionic variables, and $W(x)$ - superpotential defining the interaction. In the framework of this model, the criterion of spontaneous breaking was formulated in terms of the so called Witten index.

Very soon, it became clear that SUSY QM provides the really new approach to many problems of Quantum Mechanics itself [4], [5]. Now, it is one of the most effective approaches for analytical methods in Quantum Mechanics. The new notion of shape invariance [6] formulated in SUSY QM has to be noticed here since it allows to solve problems in a pure algebraic way. A new impetus was given to the development of multidimensional $d>1$ Quantum Mechanics [5]. In particular, before this time the most known and most effective regular approach for study of $d=2$ systems was the separation of variables. It reduces the multidimensional (multiparticle) problem to a few problems of

\footnotetext{
${ }^{\text {a} E-m a i l: ~ m . i o f f e @ s p b u . r u ~}$

${ }^{b}$ E-mail: e.v.kolev@yandex.ru

${ }^{c}$ E-mail: vvv@av2467.spb.edu
} 
lower dimensionality of space. SUSY QM provides the new way for study of 2-dim models which are not amenable to standard separation of variables: several two-dimensional models not amenable to conventional separation of variables were shown to be exactly solvable [7].

\section{SUSY Quantum Mechanics of E.Witten}

The simplest superalgebra includes three elements - superHamiltonian $\widehat{H}$ and mutually conjugate supercharges $\widehat{Q}^{+}, \widehat{Q}^{-}=\left(\widehat{Q}^{+}\right)^{\dagger}$ - with the following superalgebra:

$$
\left[\widehat{H}, \widehat{Q}^{+}\right]=\left[\widehat{H}, \widehat{Q}^{-}\right]=0 ;\left\{\widehat{Q}^{+}, \widehat{Q}^{-}\right\}=\widehat{H} ;\left\{\widehat{Q}^{ \pm}, \widehat{Q}^{ \pm}\right\}=0
$$

This algebra admit the simplest realization by $2 \times 2$ matrix differential operators in one variable:

$$
\begin{aligned}
\widehat{H} & =\left(\begin{array}{cc}
h^{(0)}(x) & 0 \\
0 & h^{(1)}(x)
\end{array}\right) ; \widehat{Q}^{+}=\left(\begin{array}{cc}
0 & 0 \\
q^{-} & 0
\end{array}\right) ; \\
\widehat{Q}^{-} & =\left(\begin{array}{cc}
0 & q^{+} \\
0 & 0
\end{array}\right)
\end{aligned}
$$

where

$$
h^{(i)}=-\partial^{2}+V^{(i)}(x), \quad q^{\mp} \equiv \pm \partial+\partial W(x) ; \quad \partial \equiv d / d x .
$$

The nilpotency of $\widehat{Q}^{ \pm}$is ensured by their matrix structure. Anticommutator of $\widehat{Q}^{ \pm}$gives $\widehat{H}$ if $h^{(0)}, h^{(1)}$ are factorized:

$$
h^{(0)}=q^{+} q^{-} ; \quad h^{(1)}=q^{-} q^{+} .
$$

This factorization ensures also the commutation of $\widehat{H}$ with $\widehat{Q}^{ \pm}$, which in components takes the form of the intertwining relations:

$$
h^{(0)} q^{+}=q^{+} h^{(1)} ; \quad q^{-} h^{(0)}=h^{(0)} q^{-},
$$

which are the most important relations of SUSY QM approach. In components, the above relations of SUSY algebra reproduce the well known Factorization Method of Schrödinger [8].

One has almost coinciding spectra of $h^{(0)}$ and $h^{(1)}$ or, equivalently, double degeneracy of the energy spectrum of $\widehat{H}$ :

$$
\begin{gathered}
h^{(0)} \Psi_{n}^{(0)}=E_{n} \Psi_{n}^{(0)} ; \Psi_{n}^{(1)}=q^{-} \Psi_{n}^{(0)} ; n=0,1, \ldots \\
h^{(1)} \Psi_{n}^{(1)}=q^{-} q^{+} q^{-} \Psi_{n}^{(0)}=E_{n} q^{-} \Psi_{n}^{(0)}=E_{n} \Psi_{n}^{(1)} \\
\Psi_{n}^{(0)}(x)=q^{+} \Psi_{n}^{(1)}(x) ; n=0,1, \ldots
\end{gathered}
$$

Although SUSY QM explores the typical terminology of supersymmetrical models in Elementary Particle Physics: supersymmetry, superpartners, boson-fermion's multiplets, supertransformations etc., the physical meaning of many terms may be very different.

\section{Shape invariance and exact solvability}

The notion of shape invariance appeared [6] for the first time in d=1 SUSY QM. This property provides very elegant approach to solve a variety of Schrödinger equations in a pure algebraic way. Let $h^{(0)}, h^{(1)}$ depend not only on $x$, but also on some parameter $a$. Let both Hamiltonians participate in 
the intertwining relations given above. Let the dependence of Hamiltonians on parameter $a$ obeys the property of "shape invariance":

$$
h^{(0)}(x ; a)=h^{(1)}(x ; \tilde{a})+R(a) ; \quad \tilde{a}=\tilde{a}(a),
$$

where the shift $R(a)$ does not depend on coordinate $x$. If the ground state of $h^{(1)}(x ; a)$ is fixed as $E_{0}^{(1)}(a)=0$, and if its zero mode is known:

$$
q^{+}(x ; a) \Psi_{0}^{(1)}(x ; a)=0,
$$

the entire spectral problem for $h^{(1)}(x ; a)$ is solved algebraically due to intertwining relations. In particular, the first excited state is:

$$
h^{(1)}(x ; a)\left(q^{-}(x ; a) \Psi_{0}^{(1)}(x ; \tilde{a})\right)=R(a)\left(q^{-}(x ; a) \Psi_{0}^{(1)}(x ; \tilde{a})\right)
$$

Analogously, the higher excited states can be built by the action of the next $q^{-}$:

$$
\begin{gathered}
h^{(1)}(x ; a)\left(q^{-}(x ; a) q^{-}(x ; \tilde{a}) \Psi_{0}^{(1)}(x ; \tilde{\tilde{a}})\right)= \\
=(R(a)+R(\tilde{a}))\left(q^{-}(x ; a) q^{-}(x ; \tilde{a}) \Psi_{0}^{(1)}(x ; \tilde{\tilde{a}})\right) .
\end{gathered}
$$

As a remark, all well known exactly solvable potentials are shape-invariant. And vice a versa, under some natural conditions on the superpotentials, shape invariant potentials give just a list of known exactly solvable models [9]. Nevertheless, the intertwining relations are the source of new solvable potentials - superpartners of the old ones.

A generalization of shape invariance in $d=1$ is based on the polynomial SUSY QM [10] the special deformation of Witten's SUSY algebra. Briefly speaking, if one'll choose intertwining operators (components of supercharge) to be of second order:

$$
q^{+} \equiv \partial^{2}+f(x) \partial+b(x)
$$

the SUSY algebra takes the same Witten's form, but the anticommutator of supercharges becomes now second order polynomial in $H$

$$
\left\{\widehat{Q}^{+}, \widehat{Q}^{-}\right\}=\widehat{H}^{2}+\alpha \widehat{H}+\beta
$$

It is important that not all such $q^{ \pm}$are factorizable, and therefore some new solutions of intertwining relations can be built. The shape invariance property with these supercharges also leads to a new class of potentials:

$$
V^{(0,1)}(x)=\frac{2 c(2 a \mp 1)}{3 x^{2 / 3}}-\frac{5}{36 x^{2}}+c^{2} x^{2 / 3} .
$$

The Schrödinger equation with this potential was solved analytically, it turned out [11] to be an implicitly solvable model.

\section{Two-dimensional generalizations of SUSY QM}

The direct multidimensional generalization was built in a series of papers [12] by another choice of realization for the elements $\widehat{H}, \widehat{Q}^{+}, \widehat{Q}^{-}$of SUSY algebra. It was made in two ways: by handmade 
extension of 1-dim formulas, and by means of the superfield approach of Quantum Field Theory. The main idea of the latter was to consider the model of scalar real multicomponent superfield:

$$
\Phi_{k}(t, \theta, \bar{\theta})=x_{k}(t)+i\left(\bar{\theta} \psi_{k}(t)+\theta \bar{\psi}_{k}(t)\right)+\frac{1}{2} \bar{\theta} \theta F_{k}(t)
$$

where the number of components $k=1,2, \ldots, d$. It was shown that in this case the superHamiltonian $\widehat{H}$ includes a chain of matrix components of the Schrödinger form but with matrix potential. The intertwining relations between matrix Hamiltonians lead to interrelations between spectra of neighbouring components of the Superhamiltonian.

Matrix Hamiltonians occur in different branches of Physics: particles with spin, coupled channels systems etc. The interesting results were obtained, such as the spectrum of Pauli operator for spin $1 / 2$ particle in a class of nonhomogeneous external electromagnetic field [13] and the relations between amplitudes and phases of $N N-$ and $N \bar{N}$-scattering in triplet and singlet states [14].

Is it possible to avoid matrix Hamiltonians from the $d$-dimensional SUSY QM ? Any attempt to intertwine two scalar Hamiltonians by means of first order operators $q^{ \pm}$leads to potentials with standard separation of variables. The polynomial SUSY QM with second order supercharges turned out to be fruitful in construction of 2-dim SUSY QM without matrix Hamiltonians at all.

Concerning solvability of two-dimensional quantum models, the only regular way is the standard separation of variables:

$$
h(\vec{x})=h_{1}\left(x_{1}\right)+h_{2}\left(x_{2}\right) ; \quad \Psi(\vec{x})=\sum c_{i k} \psi_{1 i}\left(x_{1}\right) \cdot \psi_{2 k}\left(x_{2}\right) .
$$

The complete classification of such models was given by L.P.Eisenhart [15]: separation is possible in the following coordinates - Cartesian, polar, elliptic and parabolic. The general form of potentials amenable to separation is known explicitly up to arbitrary functions of one variable.

It is very interesting to expand the list of solvable two-dimensional models beyond the class of models with separation of variables. Two-dimensional polynomial SUSY Quantum Mechanics [16] is useful to find new solvable systems. The main idea is to intertwine scalar Hamiltonians $h^{(0)}(\vec{x}), h^{(1)}(\vec{x})$ by second order operators:

$$
h^{(0)} q^{+}=q^{+} h^{(1)} ; \quad q^{-} h^{(0)}=h^{(1)} q^{-}
$$

Here, intertwined Hamiltonians have still the Schrödinger form:

$$
h^{(0,1)}=-\partial_{i}^{2}+V^{(0,1)}(\vec{x}) \text {, }
$$

but intertwining operators have the most general second order form now:

$$
q^{+}=g_{i k}(\vec{x}) \partial_{i} \partial_{k}+C_{i}(\vec{x}) \partial_{i}+B(\vec{x}) ; \quad q^{-} \equiv\left(q^{+}\right)^{\dagger}
$$

The intertwining relations are equivalent to a complicate system of nonlinear second order differential equations for functions $g_{i k}(\vec{x}), C_{i}(\vec{x}), B(\vec{x})$, and potentials $V^{(0,1)}(\vec{x})$. Finally, with some ansatzes it was reduced to the functional-differential equation, which does not admit any regular procedure of solution.

Nevertheless, some particular solutions were found for Lorentz form of metric in supercharges $g_{i k}(\vec{x})=\operatorname{diag}(1,-1)$, when the system is simplified essentially. Wide list of such pairs of potentials was found in a series of papers [16], [7]. 


\section{SUSY QM and new separation of variables: $d=2$}

The polynomial two-dimensional SUSY QM allowed to build two new procedures [17], [18], [7], [5] for solution of the Schrödinger equation which can be useful when the standard separation of variables does not work:

1) Partial solvability, or quasi-exact solvability (QES) in another terminology;

2) Complete solvablity;

Both methods were called as supersymmetrical separations of variables.

The first variant is realized when both Hamiltonians $h^{(0,1)}(\vec{x})$ DO NOT allow separation of variables, but the supercharge $q^{+}(\vec{x})$ DOES allow. In this case, the general scheme is the following. Let's suppose that we know zero modes of $q^{+}$:

$$
q^{+} \Omega_{n}(\vec{x})=0 ; \quad n=0,1, \ldots, N ; \quad q^{+} \vec{\Omega}(\vec{x})=0 .
$$

The important property of intertwining relations $h^{(0)} q^{+}=q^{+} h^{(1)}$ is that the space of zero modes is closed under the action of $h^{(1)}$ :

$$
h^{(1)} \vec{\Omega}(\vec{x})=\hat{C} \vec{\Omega}(\vec{x}),
$$

where $\hat{C}$ is the constant matrix. If this matrix can be diagonalized, i.e.

$$
\hat{B} \hat{C}=\hat{\Lambda} \hat{B} ; \quad \hat{\Lambda}=\operatorname{diag}\left(\lambda_{0}, \lambda_{1}, \ldots, \lambda_{N}\right),
$$

we obtain the set of eigenvalues of $h^{(1)}$ algebraically:

$$
h^{(1)}(\hat{B} \vec{\Omega}(\vec{x}))=\hat{\Lambda}(\hat{B} \vec{\Omega}(\vec{x}))
$$

To realize this scheme, we have to find zero modes $\Omega_{n}(\vec{x})$ and to find constant matrix $B$ diagonalizing $\hat{C}$.

For the Lorentz metric in supercharge, the special similarity (not unitary) transformation allows to separate variables in operator $q^{+}$. By this reason the following procedure is called as SUSY-separation of variables. Similarly to the standard separation, the next task is to solve two one-dimensional problems. Fortunately, for several problems this is possible. The problem of diagonalization of matrix $\hat{C}$ was solved as well by some specific procedure [17]. It is clear in advance that there is no reasons to expect exact solvability of the problem by this procedure. This is quasi-exact solvability (QES) when only a part of spectrum and corresponding wave functions can be built.

The applicability of the full scheme to the concrete models depends on analytical solvability of two one-dimensional problems. A good example - generalized 2-dim Morse potential:

$$
\begin{aligned}
V & =\alpha^{2} a(2 a \mp 1) \sinh ^{-2}\left(\frac{\alpha x_{-}}{2}\right)+ \\
& +A\left[e^{-2 \alpha x_{1}}-2 e^{-\alpha x_{1}}+e^{-2 \alpha x_{2}}-2 e^{-\alpha x_{2}}\right] .
\end{aligned}
$$

The model is called as two-dimensional Morse due to its form:

$$
V(\vec{x})=V_{\text {Morse }}\left(x_{1}\right)+V_{\text {Morse }}\left(x_{2}\right)+v\left(x_{1}, x_{2}\right) \text {, }
$$

where the first two terms have the well known one-dimensional Morse form. In this case, separation of variables in $q^{+}$leads to a pair of one-dimensional Morse problems, which are exactly solvable. The matrix $\hat{C}$ was found explicitly - it turned out to be triagonal. Therefore, the energy eigenvalues coincide with its elements. Construction of the wave functions is a much more difficult task, but it was 
solved as well [17]. Each wave function constructed by SUSY-separation produces a set of additional wave functions due to shape invariance property of the model:

$$
\begin{aligned}
h^{(0)}(\vec{x} ; a) & =h^{(1)}(\vec{x} ; \tilde{a})+\mathcal{R}(a) ; \tilde{a}=a-1 / 2 ; \\
\mathcal{R}(a) & =\alpha^{2}(4 a-1) .
\end{aligned}
$$

The same two-dimensional Morse potential is a good illustration of the second procedure of SUSY-separation of variables [18], [7], [5]. Let's suppose that, due to the specific choice of parameters of the problem, one of the intertwined Hamiltonians DOES allow the standard separation of variables, but its superpartner still DOES NOT. In our case, $h^{(1)}$ allows separation if $a=-1 / 2$, when the mixed term in potential vanishes. Due to intertwining, wave functions $\Psi^{(0)}$ of $h^{(0)}$ are obtained as usual:

$$
\Psi^{(0)}=q^{+} \Psi^{(1)} .
$$

Because of nontrivial action of supercharges, which are the second order differential operators, some additional wave functions could exist, in general. But this possibility is not realized in our case. The whole spectrum of $h^{(0)}$ and corresponding normalized wave functions were found for two-dimensional Morse potential. Due to shape invariance, again the result can be extended to all negative half-integer values of $a$.

Both procedures of of SUSY-separation of variables described above work also for twodimensional Pöschl-Teller [19] potential and for two-dimensional Scarf II [20] potential.

\section{Acknowledgements}

The work of E.V.K. was partially supported by the Saint Petersburg State University grant N 11.38.223.2015. The work of V.V.V. was partially supported by the Saint Petersburg State University grant N 11.38.189.2014.

\section{References}

[1] Y.A. Gol'fand, E.P. Likhtman, JETP Letters 13323 (1971); J.-L. Gervais, B. Sakita, Phys. Rev. D 42291 (1971); P. Ramond, Phys. Rev. D 32415 (1971); A. Neveu, J. Schwarz, Nucl. Phys. B 3186 (1971); D.V. Volkov, V.P. Akulov, Phys. Lett. B 46109 (1973); J. Wess, B. Zumino, Nucl. Phys. B 7039 (1974);

[2] S. Coleman, J. Mandula, Phys. Rev. 1591251 (1967).

[3] E. Witten, Nucl. Phys. B, 188513 (1981).

[4] F. Cooper, A. Khare, U. Sukhatme, Phys. Rep., 25268 (1995); B.K. Bagchi, Supersymmetry in Quantum and Classical Mechanics (Chapman, Boca Raton, 2001); D.J. Fernandez C, AIP Conf. Proc., 12873 (2010).

[5] A.A. Andrianov, M.V. Ioffe, J. Phys. A 45503001 (2012).

[6] L.E. Gendenshtein, JETP Lett. 38356 (1983).

[7] M.V. Ioffe, J. Phys. A 3710363 (2004); M.V. Ioffe, SIGMA 6075 (2010).

[8] L. Infeld, T.E. Hull, Rev. Mod. Phys. 2321 (1951).

[9] J. Bougie, A. Gangopadhyaya, J.V. Mallow, Phys. Rev. Lett. 105210402 (2010); J. Bougie, A. Gangopadhyaya, J.V. Mallow, J. Phys. A 44275307 (2011).

[10] A.A. Andrianov, M.V. Ioffe, V.P. Spiridonov, Phys. Lett. A 174273 (1993); A.A. Andrianov, F. Cannata, J.-P. Dedonder, M.V. Ioffe, Int. J. Mod. Phys. A 102683 (1995).

[11] F. Cannata, M.V. Ioffe, E.V. Kolevatova, D.N. Nishnianidze, Ann. Phys. 356438 (2015). 
[12] A.A. Andrianov, N.V. Borisov, M.V. Ioffe, JETP Lett. 3993 (1984); A.A. Andrianov, N.V. Borisov, M.V. Ioffe, Phys. Lett. A 10519 (1984); A.A. Andrianov, N.V. Borisov, M.V. Ioffe, Theor. Math. Phys. 611078 (1984); A.A. Andrianov, N.V. Borisov, M.I. Eides, M.V. Ioffe, Phys. Lett. A 109143 (1985); A.A. Andrianov, N.V. Borisov, M.I. Eides, M.V. Ioffe, Theor. Math. Phys. 61965 (1984).

[13] A.A. Andrianov, M.V. Ioffe, Phys. Lett. B 205507 (1988); M.V. Ioffe, A.I. Neelov, J. Phys. A 36 2493 (2003); F. Cannata, M.V. Ioffe, A.I. Neelov, D.N. Nishnianidze, J. Phys. A 3710339 (2004).

[14] A.A. Andrianov, N.V. Borisov, M.V. Ioffe, Phys. Lett. B 181141 (1986); A.A. Andrianov, N.V. Borisov, M.V. Ioffe, Theor. Math. Phys. 72748 (1986).

[15] L.P. Eisenhart, Phys. Rev. 7487 (1948); L.P. Eisenhart, Ann. Math. 35284 (1934).

[16] A.A. Andrianov, M.V. Ioffe, D.N. Nishnianidze, Phys. Lett. A 201 (1995) 103; A.A. Andrianov, M.V. Ioffe, D.N. Nishnianidze, Polynomial supersymmetry and dynamical symmatries in Quantum Mechanics Theor. Math. Phys. 1041129 (1995);

A.A. Andrianov, M.V. Ioffe, D.N. Nishnianidze, Higher order SUSY in Quantum Mechanics and integrability of two-dimensional Hamiltonians preprint solv-int/9605007 (1996); A.A. Andrianov, M.V. Ioffe, D.N. Nishnianidze, J. Phys. A 324641 (1999).

[17] F. Cannata, M.V. Ioffe, D.N. Nishnianidze, J. Phys. A 351389 (2002).

[18] M.V. Ioffe, D.N. Nishnianidze, Phys. Rev. A 76052114 (2007);

[19] M.V. Ioffe, P.A. Valinevich, J. Phys. A 382497 (2005); M.V. Ioffe, D.N. Nishnianidze, P.A. Valinevich, J. Phys. A 43485303 (2010).

[20] M.V. Ioffe, E.V. Krupitskaya, D.N. Nishnianidze, Ann. Phys. 327764 (2012); M.V. Ioffe, E.V. Krupitskaya, D.N. Nishnianidze, EPL 9810013 (2012). 